\title{
Vocabulary usage among Asian EFL learners during speech: a corpus-based quantitative analysis of the effects of L1 type, L2 proficiency and task type
}

\author{
Shin'ichiro Ishikawa \\ IPHE, Kobe University, Kobe, Japan
}

\begin{abstract}
Purpose - Using a newly compiled corpus module consisting of utterances from Asian learners during L2 English interviews, this study examined how Asian EFL learners' L1s (Chinese, Indonesian, Japanese, Korean, Taiwanese and Thai), their L2 proficiency levels (A2, B1 low, B1 upper and B2+) and speech task types (picture descriptions, roleplays and QA-based conversations) affected four aspects of vocabulary usage (number of tokens, standardized type/token ratio, mean word length and mean sentence length).

Design/methodology/approach - Four aspects concern speech fluency, lexical richness, lexical complexity and structural complexity, respectively.

Findings - Subsequent corpus-based quantitative data analyses revealed that (1) learner/native speaker differences existed during the conversation and roleplay tasks in terms of the number of tokens, type/token ratio and sentence length; (2) an L1 group effect existed in all three task types in terms of the number of tokens and sentence length; (3) an L2 proficiency effect existed in all three task types in terms of the number of tokens, type-token ratio and sentence length; and (4) the usage of high-frequency vocabulary was influenced more strongly by the task type and it was classified into four types: Type A vocabulary for grammar control, Type B vocabulary for speech maintenance, Type $\mathrm{C}$ vocabulary for negotiation and persuasion and Type $\mathrm{D}$ vocabulary for novice learners.

Originality/value - These findings provide clues for better understanding L2 English vocabulary usage among Asian learners during speech.
\end{abstract}

Keywords Spoken learner corpus, L2 English vocabulary use, Contrastive interlanguage analysis, L1 group effect, L2 proficiency effect, Speech task types

Paper type Research paper

\section{Introduction}

L2 English learners' vocabulary usage in speech may be influenced by their L1s (Götz, 2019), L2 proficiency levels (Ishikawa, 2020) and task types (Gilanlioglu, 2002; Teng, 2007; Hsieh and Wang, 2019; Guerrero, 2004). In speech, learners often consume much of their attention resources while simultaneously processing listening and speaking, thus leading to weakened L2 monitors and deviant L2 vocabulary usage.

Many previous studies have examined L2 outputs among advanced learners mainly in Europe in order to discuss how L1 transfer influences L2 vocabulary usage (Granger, 1998). However, there is a lack of focus on how Asian learners with different L1 backgrounds and L2

(C) Shin'ichiro Ishikawa. Published in PSU Research Review. Published by Emerald Publishing Limited. This article is published under the Creative Commons Attribution (CC BY 4.0) licence. Anyone may reproduce, distribute, translate and create derivative works of this article (for both commercial and noncommercial purposes), subject to full attribution to the original publication and authors. The full terms of this licence may be seen at http://creativecommons.org/licences/by/4.0/legalcode

This work was supported by JSPS KAKENHI (Grants: Number 17H02360 and Number 20H01282).

Vocabulary usage among Asian EFL learners
Received 11 January 2021 Revised 22 May 2021 15 August 2021

Accepted 13 October 2021 
proficiency levels adopt vocabulary during various types of speech tasks. This is partly due to the lack of an appropriate data set.

Thus, this study used the ICNALE Spoken Dialogue Module (Ishikawa, 2019), a recently released corpus consisting of the outputs of Asian learners during L2 English interviews, in order to investigate how learners' L1s (Chinese, Indonesian, Japanese, Korean, Taiwanese and Thai), their L2 proficiency levels (A2, B1 low, B1 upper and B2+) and speech task types (picture descriptions, roleplay and QA-based conversations) affect four aspects of vocabulary usage (number of tokens, standardized type/token ratio, mean word length and mean sentence length). This study also examined the factors that most strongly affect vocabulary usage among Asian learners.

\section{Literature review}

\subsection{Vocabulary in speech}

Vocabulary plays a vital role in successful L2 speech. Skehan (2009) emphasized the need to add lexis as a fourth dimension to the so-called complexity, accuracy and fluency (CAF) model, which is a widely adopted framework for discussing the quality of various types of speech. De Jong et al. (2012) examined the relationship between linguistic knowledge (vocabulary and grammar), linguistic processing skills (reaction time) and pronunciation skills of L2 Dutch learners, thus revealing that L2 speaking proficiency included the element of lexis. Focusing on L2 Chinese learners, Liu (2020) examined the relationship between vocabulary size, lexical access and CAF qualities in speech, thereby revealing that vocabulary size was related to all CAF measures, though the lexical retrieval speed was not directly related to syntactic complexity. Foster (2020) surveyed five kinds of canonical studies aimed at the construct of L2 fluency, showing that four discussed the relationships between variables such as vocabulary size, fluency (uttered/perceived), idiomaticity, task familiarity and learner self-reflection.

This raises the important question of what aspect of vocabulary usage is most closely related to speech quality. As shown below, many previous studies have focused on fluency and lexical richness. With regard to fluency, there has been extensive research on speed rate (i.e. the number of words uttered within a certain period). Although different methods can be used to count fluency (Segalowitz, 2010, p. 6), speed rate is usually regarded as one of the most important factors for explaining good speech. Further, speech rate is naturally related to L2 proficiency. Based on an analysis of a learner interview corpus, Ishikawa (2020) demonstrated that the speech rate of upper-intermediate learners was $40-130 \%$ higher than that of novice learners.

Meanwhile, Götz (2013) examined lexical richness, noting that native speakers were able to use words across large vocabulary sets, while learners only used those within relatively limited ranges, as they had not yet obtained all connotations and usage restrictions for individual words. In this regard, they had less confidence and experienced more difficulty when attempting to use a variety of L2 vocabulary. Thus, learners "tend to avoid supposedly 'difficult' words and constructions that require a lot of processing effort"; instead, they repeatedly use "the same, easily retrieved and 'safe' words" (p. 65), which leads to lower lexical richness/diversity.

Lexical richness is usually regarded as a reliable index of individual vocabulary size; it should be related to various aspects of learner speech. However, Mairano and Santiago (2020) examined the relationship between receptive/productive vocabulary size and pronunciation quality among L2 French learners, finding that the indices of lexical richness (which they referred to as lexical diversity) were not strongly correlated with receptive vocabulary indices or learner self-evaluations in four skills.

\subsection{Influences on learners' vocabulary usage}

For learners, aspects of L2 vocabulary usage are highly variable in speech and may be influenced by many parameters. Thus, many studies have focused on the possible effects of speech modes, learner L1s and speech tasks. 
2.2.1 Speech modes. Learners often use vocabulary quite differently in monologues and dialogues. Bell (2003) compared the fluency (speech rate) of L1 Brazilian learners of English in both regards, finding increases in dialogues when compared to monologues.

Van Os et al. (2020) emphasized the need to discuss learner proficiency in the context of interaction because "natural communication usually occurs in conversations" (p. 1183). Here, they specifically investigated how aspects of conversational turn-taking affected perceived fluency among both native and non-native speakers by artificially manipulating the speech rate and the length of pauses between questions and answers. When implemented by learners, longer pauses created the impression that answers were more reluctant, thereby resulting in lower perceived fluency.

Williams (2020) analyzed perceived fluency of learners. By examining teacher judgments, they showed that perceived fluency entailed two separate components: individual (monologue) fluency and conversational (dialogue) fluency. Here, the former pertained to temporal measures of within-clause pause rates, while the latter pertained to measures of filled-pause rates.

2.2.2 Learner L1s. For learners, the quantity and quality of vocabulary usage are both expected to vary with their L1s. Ishikawa (2020) compared the fluencies of Asian learners with different L1 backgrounds, finding that Indonesian learners spoke $12-13 \%$ more than Japanese and Korean learners in the context of picture description tasks.

Nevertheless, the L1 influence requires careful consideration. It may appear that learner fluency is directly influenced by L1s. However, scrutinizing the relationships between the number of filled pauses as disfluency markers and varied linguistic and non-linguistic elements, Götz (2019) revealed that, regardless of the L1 background, the number of filled pauses was correlated with many non-linguistic factors, including the length of English learning, the experience of staying in English-speaking countries, the relationship with the interviewer and the interviewer's gender, though it was not correlated with factors such as the interview topic or learner's gender. Ultimately, Götz (2019) suggested the importance of drawing a line "between L1-specific and universal learning features."

2.2.3 Task types. For learners, vocabulary usage may also be affected by the type of speech task. Gilanlioglu (2002) examined the effects of task conditions ( \pm planning time, descriptive/ narrative) on fluency, lexis (word range, lexis-to-grammar ratio, lexical density, lexical choice, syllabic range, lexical strategy use and lexical stretching) and grammar (complexity and accuracy) in L2 speech, thus showing interdependencies between lexis and grammar in addition to interactions between task and contextual features.

Teng (2007) analyzed speech among Taiwanese learners of English in order to clarify the effects of three task types (answering recorded questions, picture descriptions and presentations) on oral performance (rating, accuracy, complexity and fluency). Analyses showed that both complexity and fluency were significantly improved during the Q\&A tasks. The same study also assessed how participants perceived these speech tasks, revealing a general preference for picture description tasks and finding that the Q\&A and presentation tasks were seen as the most reliable ways of measuring oral skills.

Hsieh and Wang (2019) analyzed speech among young learners to clarify the relationship among fluency, grammar, vocabulary and content. More specifically, over 20 discourse features were compared across two task types (picture narration and integrated listening/ speaking) and four proficiency levels. The analysis showed that proficiency levels strongly influenced the output features, while the task types had some effects on grammar, vocabulary and content, but not on fluency.

Guerrero (2004) conducted an experiment to analyze how task complexity ( \pm planning time and \pm here-and-now context) influences learner output variables such as fluency (unpruned/pruned speech rate), lexical complexity (lexical/function word ratio and lexical richness), structural complexity ( $S$-nodes per $T$-unit) and accuracy (error-free $T$-units, target-
Vocabulary usage among Asian EFL learners 
like article usage, self-repairs and repair/unrepaired errors). The analysis showed that the planning time significantly affected both fluency and lexical complexity but did not affect structural complexity and accuracy. Findings ultimately showed that task complexity should be regarded as "an important construct for task design and task sequencing" (Guerrero, 2004).

As summarized above, the current literature provides many noteworthy facts about the major aspects of L2 English vocabulary usage among learners. However, it is rather difficult to integrate these findings, as individual studies have implemented unique data sets and analytical frameworks. In this regard, further investigation is needed to determine the mixed effects of L1s, L2 proficiency levels and speech task types on the facets of vocabulary usage among Asian learners.

\section{Research design}

\subsection{Aim and research questions}

Previous studies have examined learners' vocabulary usage in speech from many angles, but the performance of Asian EFL learners has not been fully investigated. This study investigated the ways in which and the degree to which L1s, L2 proficiency levels and speech task types influence four kinds of lexical indices among Asian learners, with an additional focus on how different learner groups could be classified in terms of high-frequency word usage and which factor plays a key role in this classification.

The following research questions (RQs) were first established:

$R Q 1$. What differences exist between Asian EFL learners and English native speakers (ENS) in terms of the four kinds of lexical indices? (Learner/ENS Gap).

$R Q 2$. What differences exist between Asian EFL learners with different L1 backgrounds in terms of the four kinds of lexical indices? (L1 Group Effect).

RQ3. What differences exist between Asian EFL learners with different L2 proficiency levels in terms of the four kinds of lexical indices? (L2 Proficiency Effect).

RQ4. (a) How can Asian EFL learners be classified in terms of high-frequency word usage? (b) Among L1s, L2 proficiency levels and task types, which factor influences these classifications most strongly? (Classification).

\subsection{Data}

This study used the ICNALE Spoken Dialogue Module (ICNALE SD; Ishikawa, 2019), which has newly been added to the International Corpus Network of Asian Learners of English. The ICNALE SD includes utterances from English interviews (approximately $40 \mathrm{~min}$ in length) that were conducted with learners from 10 countries and areas in Asia as well as English native speakers.

The interviews consisted of 10 English speech tasks ([1] to [10]) and subsequent L1 talks ([11]). Tasks (A) and (B) were related to the ICNALE common topics: a part-time job for college students and non-smoking at restaurants, respectively. The 10 English tasks were classified into three types: conversations (CV), picture descriptions (PD) and roleplays (RP) (see Table 1).

The three types of English tasks were expected to elicit different types of lexical outputs from learners. Here, their L2 monitoring levels were expected to be the highest during the PD tasks, in which they could concentrate solely on describing what they saw without worrying about what to say or how to interact with the interviewers. The second-highest levels were expected during the $\mathrm{CV}$ tasks, in which learners thought about ways to answer the questions posed to them by the interviewers. Finally, the lowest levels were expected to be found for the 


\footnotetext{
\# Task

[1] Introduction (icebreaking)

An interviewee answers easy questions about his/her English learning

[2] Picture description (A)

The interviewee describes six serial pictures about a boy having a part-time job at a computer shop to earn money to go swimming with his friends

[3] Picture description (A)-related $Q A$

The interviewee answers questions about the contents of the pictures (swimming and computers) and gives an opinion on the college students' use of smartphones

[4] Roleplay (A)

The interviewee plays the role of a college student wishing to continue his/her part-time job. The interviewee is told to persuade his/her supervisor, who firmly believes that students should not have part-time jobs, to allow him/her to continue working

[5] Roleplay (A)-related QA

The interviewee answers questions related to the topic of the roleplay (part-time jobs) and gives an opinion on the college students' part-time jobs

[6] Picture description (B)

The interviewee describes six serial pictures about a mother with her son, who tells a nearby smoker to stop smoking in the park

[7] Picture description (B)-related $Q A$

The interviewee answers questions about the contents of the pictures (a park and the depicted woman) and gives an opinion about the cleanness of public parks

[8] Roleplay (B)

The interviewee plays the role of a customer who had a meal with his/her friend at a restaurant that allows smoking. The interviewee is told to persuade a restaurant owner to refund his/her money because his/her friend could not enjoy the meal due to too much smoking

[9] Roleplay (B)-related QA

The interviewee answers questions related to the topic of the roleplay (restaurants) and gives an opinion on the ban on smoking at restaurants

[10] L2 Reflection

The interviewee answers questions about the whole interview

[11] L1 Reflection

The interviewee answers questions about each of the tasks in the interview in his/her L1
}

Vocabulary usage among Asian EFL learners

$\mathrm{CV}$

$\mathrm{RP}$

$\mathrm{CV}$

PD

$\mathrm{CV}$

$\mathrm{RP}$

$\mathrm{CV}$

$\mathrm{CV}$

Table 1

ICNALE SD interview structure

RP tasks, in which learners needed to take the initiative in negotiations; these were the most demanding communicative tasks, as learners had to persuade others to accept their requests.

\subsection{Subjects}

Although the ICNALE SD collected utterance data from college students (including graduate students) in six English as a Foreign Language (EFL) and four English as a Second Language (ESL) regions in addition to ENS, this study focused only on EFL learners and ENS.

In this study, all participants were classified into four proficiency bands linked to the Common European Framework of Reference for Languages (CEFR), as follows: A2 (Waystage), B1 Low [B1_1] (Threshold-Low), B1 Upper [B1_2] (Threshold-High) and B2+ (Vantage + ). These level classifications were based on scores from either standardized English proficiency tests (e.g. TOEFL, TOEIC or IELTS) or vocabulary size tests.

This study analyzed 405 EFL learners and 20 ENS, as shown in Table 2.

\subsection{Methods}

As a methodological framework, this study used the revised contrastive interlanguage analysis model (CIA2; Granger, 2015). The original version of the CIA (Granger, 1996) consisted of two types of comparisons, one between a given native language (NL) and interlanguage (IL) and one between interlanguages with different L1 backgrounds. However, the CIA2 emphasizes increased attention on possible variances within each of the constructs of a given native language (which is redefined as a "reference language") and interlanguage. 


\begin{tabular}{llccrrr}
\hline PRR & L1 & A2 & B1 low & B1 upper & B2+ & Sum \\
\cline { 3 - 6 } & Chinese (CHN) & 2 & 13 & 17 & 18 & 50 \\
& Indonesian (IDN) & 6 & 6 & 16 & 2 & 30 \\
& Japanese (JPN) & 31 & 29 & 28 & 12 & 100 \\
& Korean (KOR) & - & 7 & 70 & 40 \\
& Thai (THA) & 11 & 7 & 19 & 2 & 50 \\
& Taiwanese (TWN) & - & - & - & - & 20 \\
& English (ENS) & 65 & 91 & 173 & 76 & 425 \\
Table 2. & Total & Note(s): (1) The module did not include data from Korean learners at the A2 level. (2) This study discussed \\
Number of study & learners in mainland China and Taiwan separately, although their L1s were nearly identical \\
subjects &
\end{tabular}

Thus, the revised framework entails multilayered comparisons between reference-language variables (including register and dialect variables) and interlanguage variables (including task and learner variables). This study was also based on multilayered comparisons in terms of L1s, L2 proficiency levels and task types.

First, four lexical indices were calculated for each of the 72 data sets (five learner groups $\times$ four levels $\times$ three tasks $=60$; one learner group $(\mathrm{KOR}) \times$ three levels $\times$ three tasks $=9$; one ENS group $\times$ one level $\times$ three tasks $=3$ ). These included the number of tokens $(\mathrm{TKN})$, standardized type-token ratio (STTR), mean word length (MWL) and mean sentence length (MSL).

Each of the above indices is related to different aspects of L2 English vocabulary usage. More specifically, TKN is directly related to fluency (speech rate), STTR suggests the width of the vocabulary, MWL shows the difficulty or morphological complexity of the vocabulary and MSL is related to the lexical and syntactic sentence complexity.

TKN is the value per person. STTR is the value adjusted by averaging the TTR values obtained from 100-word text subsets. MWL and MSL are based on the average number of letters in one word and the average number of words in one sentence, respectively.

First, to address RQ1 (Learner/ENS Gap), the values obtained from different learner groups were converted into means and then compared to values from the ENS group. We recognized a gap only when the learner value differed from the ENS value by $10 \%$ or more.

Second, to address RQ2 (L1 Group Effect), the values obtained from four proficiency-based subgroups (only three for Korean learners) were converted into means and then converted into ratios (\%) in relation to values from the ENS group. Effects were discussed only when differences between the minimum and maximum ratios reached $10 \%$.

Third, to address RQ3 (L2 Proficiency Effect), the values obtained from learners in six regions were converted into means and then converted into ratios $(\%)$ in relation to values from the A2 level learners. The values from A2 level Korean learners were not available in the corpus and thus were substituted with those from Korean learners at an adjacent level. Effects were discussed only when differences between the A2 level and B2+ level reached 10\%.

Finally, to address RQ4 (Classification), a correspondence analysis was conducted on the frequency table containing 72 data sets (Item 1) and the top 50 most frequent words (Item 2; see Table 3).

Top 50 most frequent words

Table 3.

List of words used in the correspondence analysis
I, the, to, and, a, it, is, in, so, you, my, that, because, yes, think, can, like, have, s, t, yeah, of, for, but, we, not, he, with, time, they, there, don, or, me, very, more, if, are, this, some, do, when, just, was, people, want, go, no, one, know

Note(s): (1) “s," "t," and “don” are parts of abbreviations. (2) Fillers and topic-related nouns were excluded 
As a multivariate approach, correspondence analysis is typically used to visualize relationships between qualitative or categorical variables. Similar to principal component analysis, it can identify several dimensions (factors) that maximize correlations between items 1 and 2. Accordingly, items 1 and 2 are plotted on a single two-dimension graph (i.e. scatterplot) based on the top two dimensions, in which variables with similar qualities are positioned near each other. This clarifies how different variables can be classified as well as how items 1 and 2 are interrelated.

\section{Results and discussion}

\subsection{RQ1 Learner/ENS gap}

First, we discuss the differences between Asian EFL learners and English native speakers (see Figures 1-4).

The learner/ENS gaps are shown in Table 4. We pay attention to the cases where the learner value differed from the ENS value by $10 \%$ or more, which are shown in italics in the table.

Regarding TKN, which usually concerns oral fluency, gaps were confirmed in CV and RP. However, this was not clearly seen in PD. It thus appears that learners could not speak sufficiently during interactions but were able to do so during monologue tasks. This is likely because when doing the PD task, they had been given ample time and did not need to think about what to say or how to control interactions with the interviewer. Although this finding seems to contradict a previous observation that learner fluency increased in dialogues over monologues (Bell, 2003), such a discrepancy was presumably due to differences in both the learner backgrounds (Bell's study focused on Brazilian learners) and task designs.

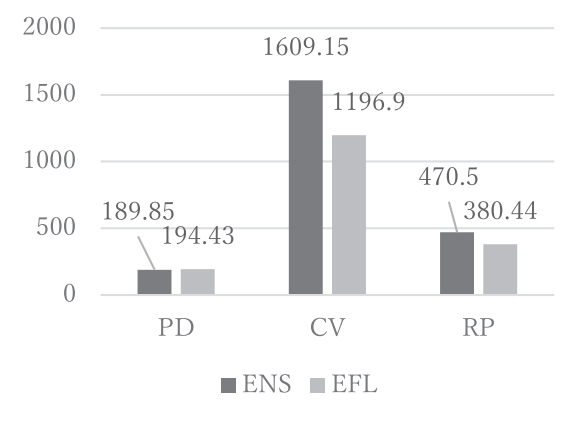

Figure 1.

TKN index for EFL learners and ENS

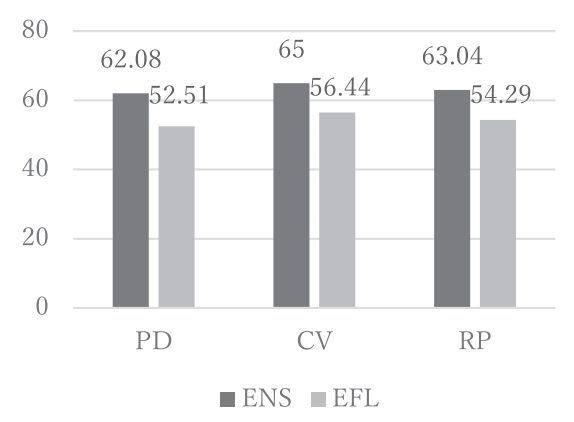

Figure 2. STTR index for EFL learners and ENS 


\section{PRR}

\section{Figure 3.}

MWL index for EFL learners and ENS

\section{Figure 4.}

MSL index for EFL learners and ENS
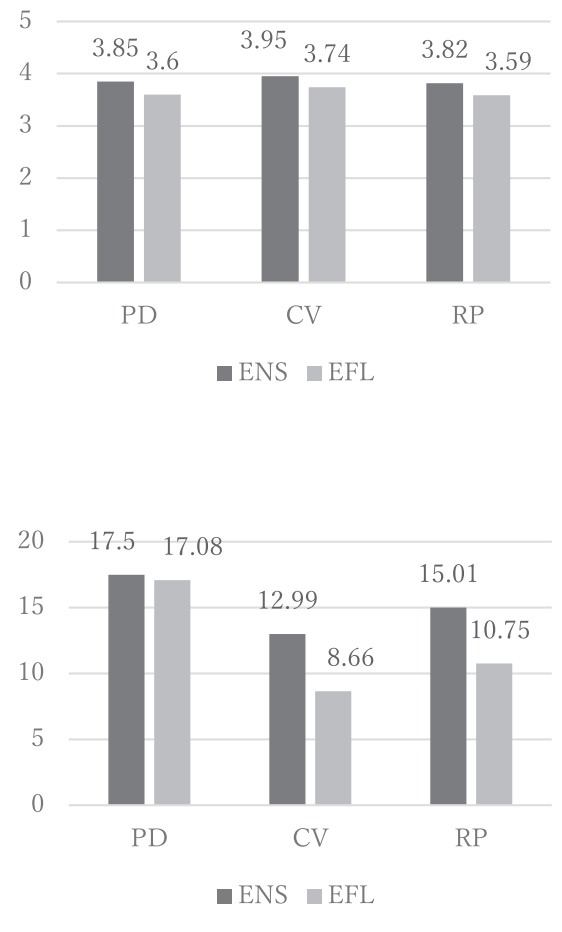

Table 4.

The learner/ESL gaps

\begin{tabular}{lcccc}
\hline Task & TKN & STTR & MWL & MSL \\
\hline PD & $4.58(2.41 \%)$ & $9.57(15.42 \%)$ & $0.25(6.49 \%)$ & $0.42(2.40 \%)$ \\
CV & $412.25(25.62 \%)$ & $8.56(13.17 \%)$ & $0.21(5.32 \%)$ & $4.33(33.33 \%)$ \\
RP & $90.06(19.14 \%)$ & $8.75(13.88 \%)$ & $0.23(6.02 \%)$ & $4.26(28.38 \%)$ \\
\hline
\end{tabular}

These findings may illuminate an inherent duality in learner fluency. If needed, they could speak sufficiently, as during the PD task; if unneeded, they chose to speak less, thus avoiding errors. In this sense, the seeming "disfluency" among learners when compared to ENS should be understood as due to not only a lack of speaking skills but also a lack of confidence and motivation, which MacIntyre et al. (1998) related to the construct known as "willingness to communicate" (WTC) in the L2 context.

Regarding STTR, which concerns lexical richness, gaps were confirmed in all three task types. This finding supports previous studies showing that learners preferred to use sets of easily retrieved "safe" words (Götz, 2013).

In terms of MWL, which concerns lexical complexity, the differences were relatively small; no clear gaps were seen in any task types. In speech, both learners and ENS seemed to use more manageable vocabulary sets.

Finally, regarding MSL, which reflects syntactic complexity, gaps were seen in CV and $\mathrm{RP}$, but were not clearly confirmed in PD. Learners could not produce sentences of sufficient durations during interactions, when their attention was largely devoted to simultaneously 
listening and speaking. However, this was not a problem during monologues, in which they were given ample time, meaning they could speak at a comfortable pace without worrying about their turns being taken by interlocutors. While Guerrero (2004) reported that the duration of planning time did not directly influence structural complexity, our data suggest the possibility that the length of learner speech varied between monologues and dialogues.
Vocabulary usage among Asian EFL learners

\subsection{RQ2 L1 group effect}

We now discuss the differences between Asian learners with different L1 backgrounds. The values shown below were all adjusted to ratios (\%) in relation to those of the ENS (see Figures 5-8).

Table 5 lists the learner groups with the highest and lowest values and also shows the differences between those values. We discuss the cases where the difference between the $\max / \mathrm{min}$ values reached $10 \%$, which are shown in italics in the table.

First, regarding TKN, L1 group effects were confirmed in all three task types. For learners, oral fluency changed to some extent across the L1-based groups. For example, IDN and CHN learners spoke more, while TWN, JPN and THA learners spoke less. However, because the values for CHN and TWN learners were not necessarily equal, the L1 group effect was not necessarily equal to the linguistically defined L1 effect. The effect observed here may be attributed to either individual learning backgrounds, as suggested by Götz (2019), or the social and educational backgrounds of learner groups from different countries and regions.

Next, regarding STTR, effects were seen in both PD and RP but not in CV, although the difference between task types was very small. In all task types, KOR learners used a slightly wider range of vocabulary, while JPN learners depended on a narrower range than the other learner groups in Asia.

Regarding MWL, differences between max/min values were small and no apparent L1 group effects were observed. Unlike other lexical indices, the MWL may be less likely to vary. Despite differences in L1 and educational background, this suggests that Asian EFL learners consistently depended on a more accessible set of vocabulary, which may be interpreted as a

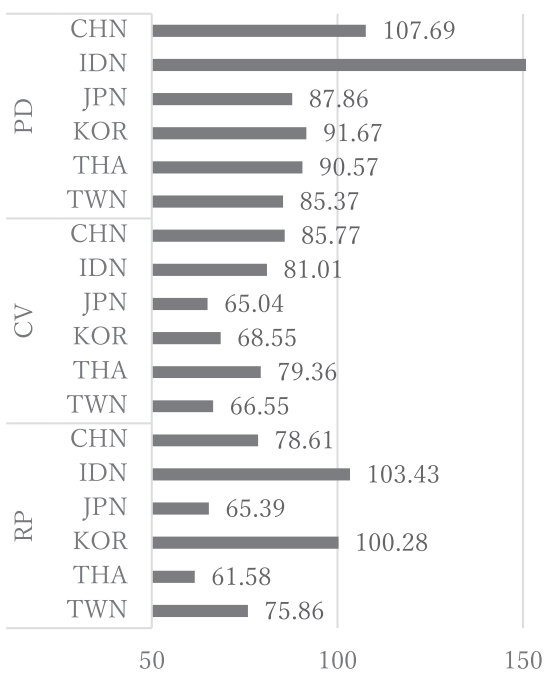

Figure 5. 


\section{PRR}

Figure 6.

STTR ratio for EFL learners

Figure 7.

MWL ratios for EFL learners
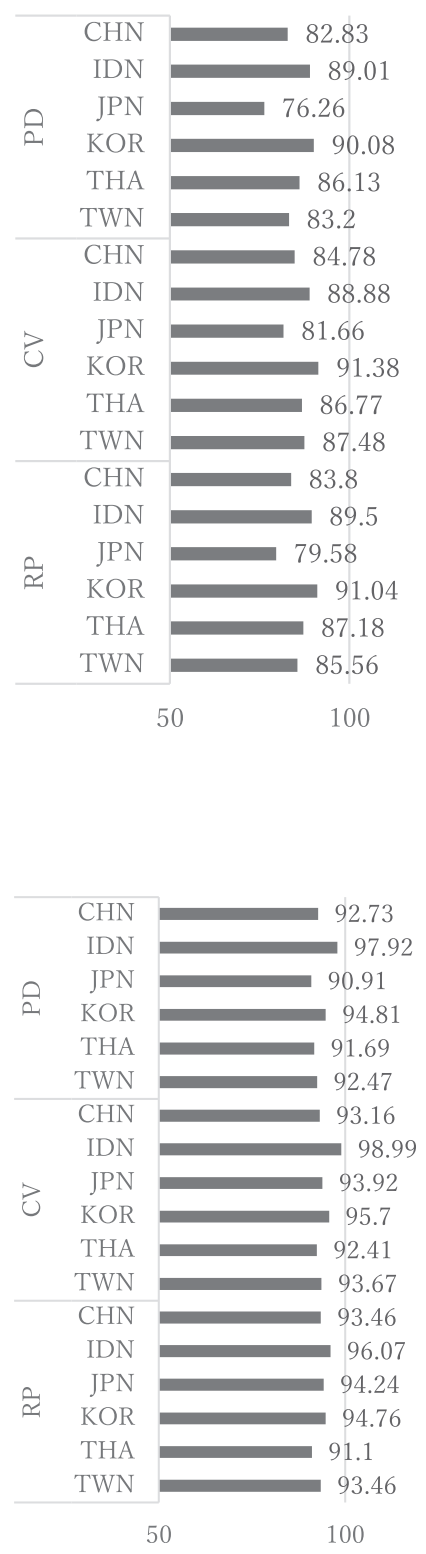

150

communicative strategy designed to compensate for the lack of lexical knowledge while maintaining speech production. Here, it is important to note that IDN learners, who spoke the most during the PD and RP tasks, tended to use slightly more complex words than other learners. In this regard, they seemed to be more active communicators with higher levels of motivation for speech development. 


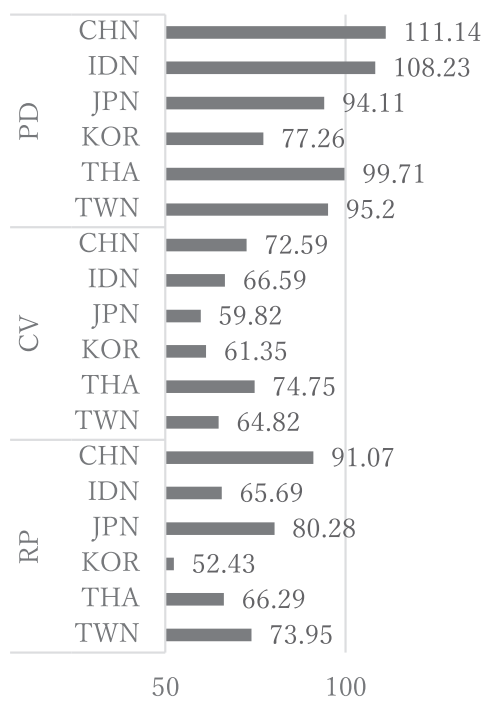

Vocabulary usage among Asian EFL learners

Figure 8. MSL ratio for EFL learners

\begin{tabular}{|c|c|c|c|c|}
\hline Task & TKN & STTR & MWL & MSL \\
\hline \multirow[t]{3}{*}{ PD } & [+] IDN (151.30) & {$[+]$ KOR (90.08) } & [+] IDN (97.92) & {$[+] \mathrm{CHN}(111.14)$} \\
\hline & {$[-]$ TWN (85.37) } & {$[-] \mathrm{JPN}(76.26)$} & [-]JPN (90.91) & {$[-] \mathrm{KOR}(77.26)$} \\
\hline & Dif: 65.93 & Dif: 13.82 & Dif: 7.01 & Dif: 33.88 \\
\hline \multirow[t]{3}{*}{$\mathrm{CV}$} & {$[+]$ CHN (85.77) } & [+] KOR (91.38) & [+] IDN (98.99) & [+] THA (74.75) \\
\hline & {$[-] J P N(65.04)$} & {$[-] J P N(81.66)$} & [-] THA (92.41) & {$[-] J P N(59.82)$} \\
\hline & Dif: 20.73 & Dif: 9.72 & Dif: 6.58 & Dif: 14.75 \\
\hline \multirow[t]{3}{*}{$\mathrm{RP}$} & [+] IDN (103.43) & [+] KOR (91.04) & {$[+] \mathrm{IDN}(96.07)$} & {$[+] \mathrm{CHN}(91.07)$} \\
\hline & [-] THA (61.58) & [-]JPN (79.58) & [-] THA (91.10) & {$[-]$ KOR (52.43) } \\
\hline & Dif: 41.85 & Dif: 11.46 & Dif: 4.97 & Dif: 38.64 \\
\hline
\end{tabular}

Table 5. The gap between learner groups showing the highest and lowest values

Finally, regarding MSL, effects were confirmed for all three task types. Results clearly showed that sentence length and conversational turn duration varied across the L1-based learner groups. CHN and THA learners produced longer sentences, whereas KOR and JPN learners produced shorter sentences. Despite this, KOR learners still used a more comprehensive range of vocabulary, as mentioned earlier.

\subsection{RQ3 L2 proficiency effect}

This section discusses the differences between Asian learners at different L2 proficiency levels. The values shown below were all adjusted to ratios $(\%)$ related to values from novice learners at the A2 level (see Figures 9-12).

We saw increasing trends for all four indices, which seems to support Hsieh and Wang (2019), who reported that proficiency levels played more significant roles than task types with regard to grammar and lexis usage. The L2 proficiency effects are shown in Table 6. We discuss the cases where the gap between A2 and B2+ learners reached $10 \%$, which are shown in italics. 
First, regarding TKN, effects were confirmed for all three task types. More advanced learners spoke more than novice learners. The degree of the increase was the highest in RP. The proficiency gap seemed to be more salient during cognitively challenging tasks.

Figure 9.

Change in TKN ratios

Figure 10.

Change in STTR ratios

Figure 11.

Change in MWL ratios

Figure 12.

Change in MSL Ratios
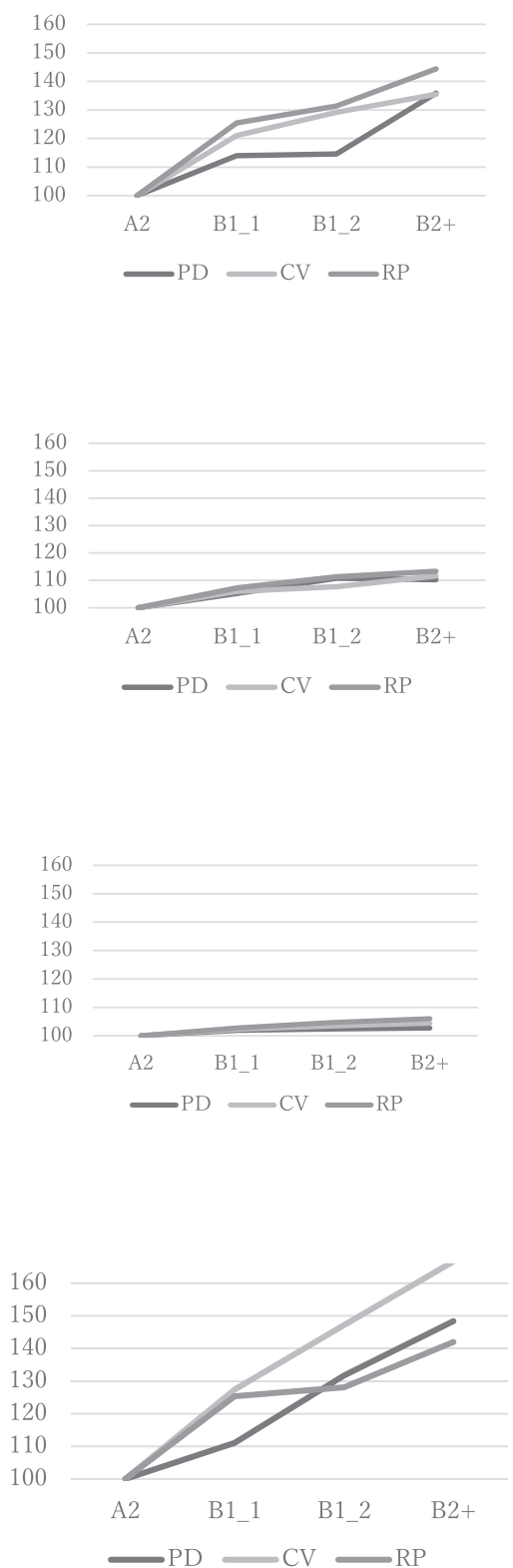
Below are quotes from the novice (1-2) and upper-intermediate (3-4) learners, both answering the question: "Why do you like to speak in English?" given during the introductory Q\&A session as a part of $\mathrm{CV}$.

(1) I... I like a foreign country and I want to .... I want to be a job uh to use English (JPN_044_A2)

(2) I think it's - is a challenger for me to speak for other people (TWN_027_A2)

(3) So, I think English is good communication to - to - to communicate with a lot of people, including the foreign people, so communicating with a lot of people; we can know a lot of information and prepare our - our interesting area (JPN_002_B2+)

(4) Uh, uh, because uh, because I read a lot in English, so uh, uh, speaking in - speaking in English helps me think more efficiently [Interviewer: Efficiently? Uh-huh, why so?] Uh, because, uh, while reading the text, you have to translate into Chinese and then, thinking in Chinese and then, translate into English again. So, uh, speaking English like, it helps me understand the text more (TWN_001_B2)

These examples illustrate how their utterances developed both quantitatively and qualitatively between the A2 level and B2+ level, although many language problems remained, even in utterances from $\mathrm{B} 2+$ learners.

Next, with regard to STTR, the widths of the gaps were much smaller but effects were observed in all three task types. More advanced learners spoke with a greater variety of vocabulary. Although the proficiency level classification in the ICNALE SD was based on scores from the receptive and knowledge-based tests, proficiency levels measured thus were proven to correlate well with the lexical quality of their oral performance.

Meanwhile, regarding MWL, the width of the gap was small. No clear proficiency effect was confirmed. This is presumably because both the novice and advanced learners tended to rely on simple/short words, even if they knew longer and more complex words. Hasselgren (1994) previously referred to such a set of easily retrieved words as a "lexical teddy bear."

Finally, with regard to MSL, effects were observed in all three task types, with the increase reaching $66.7 \%$ in $\mathrm{CV}$. In this task, novice learners tended to provide the shortest answers to the given questions, while advanced learners often added information to make their answers more meaningful.

\subsection{RQ4 Classification}

Finally, we discuss how Asian learners with different L1 backgrounds and L2 proficiency levels can be classified in terms of high-frequency word usage while performing different task types. We also discuss which of the L1 group effect, L2 proficiency effect and task effect played the most critical role in these classifications.

As mentioned, we conducted a correspondence analysis based on the frequency table, including 72 data sets as Item 1 and the top 50 most frequent words as Item 2 . The results are shown in the scatterplot below.

The contribution of Axis 1 (horizontal axis) was $59.9 \%$ while that of Axis 2 (vertical axis) was $11.2 \%$, meaning this data set was first divided into left and right halves and then into upper and lower halves.
Vocabulary

usage among

Asian EFL

learners

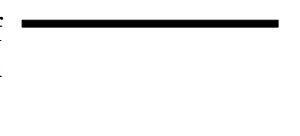




\section{PRR}

Figure 13.

Scatter plot based on the correspondence analysis
First, we investigate the key factor that determined the classification of this data set. If a speaker's L1 is the most important factor in data classification, then variables belonging to the same L1 groups should be jointly classified in either a right half or a left half. Likewise, if a speaker's L2 proficiency is the key factor, variables of the same L2 proficiency levels should be jointly classified; if a task type is the key, then variables of the same task types should be jointly clustered.

As shown in Figure 13, all variables positioned in the left half are related to $\mathrm{RP}$ or $\mathrm{CV}$, while all those positioned in the right half are related to PD. We then examine the vertical classification. In the right half, no clear differences were observed between the upper and lower halves, while in the left half, all variables positioned in the upper half are related to CV; most of those positioned in the lower half are related to RP. These observations indicate that the speech task type played the key role in the classification of the current data set.

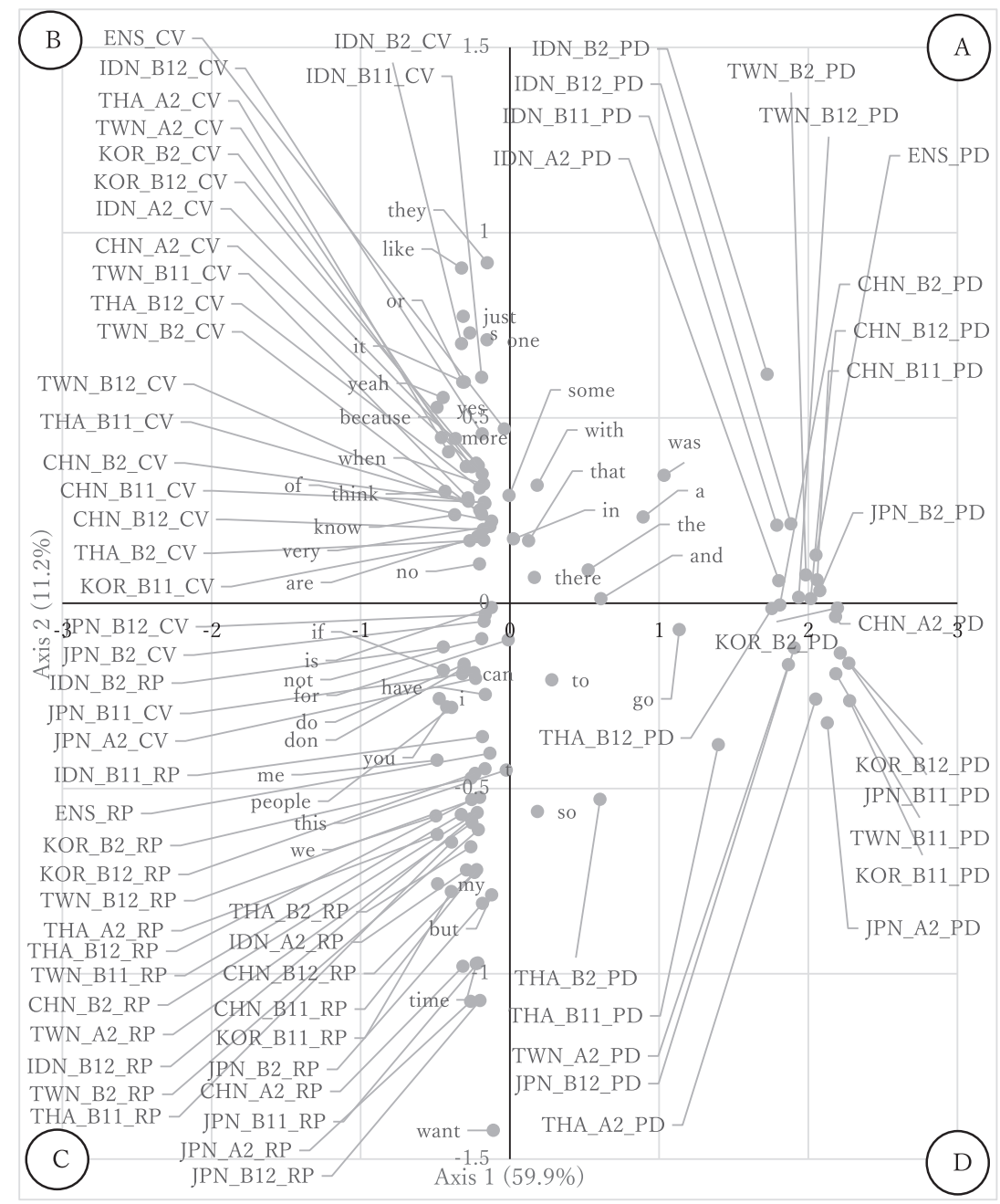


With Axis 1 and Axis 2, the 72 data sets were classified into four quadrants: $\mathrm{A}(\mathrm{X}:+, \mathrm{Y}:+)$, $\mathrm{B}(\mathrm{X}:-, \mathrm{Y}:+), \mathrm{C}(\mathrm{X}:-, \mathrm{Y}:-)$ and $\mathrm{D}(\mathrm{X}:+, \mathrm{Y}:-)$. These four quadrants represent four patterns in L2 vocabulary usage during interviews, which we refer to as Types A through D (see Table 7).

As mentioned above, the four types were determined mainly in terms of the task type. Meanwhile, regarding L2 proficiency as well as L1, many items were mixed, with no clear patterns. Here, it should be noted that the three types of ENS data were scattered across three quadrants (A, B and C), which also shows that $\mathrm{L} 1$ was not a decisive factor in classifying the current data set.

4.4.1 Type A vocabulary (for grammar control). Type A vocabulary includes a set of words mainly used in the PD task by CHN, IDN and TWN learners at the intermediate and advanced levels. It is characterized by the following words: $(1)$ articles $(a$, the $)$, (2) prepositions (in, with), (3) conjunctions (and, that), (4) past-tense verbs (was) and (5) existential there (there). The frequent adoption of these words usually suggests a higher level of grammatical control. In PD, learners could speak at a comfortable pace while conserving attention resources. Here, upper-level learners seemed to monitor their own L2 outputs by paying sufficient attention to the number of nouns, verb tenses and semantic unit connections.

This brings us to an utterance from an IDN learner during the PD task, where we requested the participants to begin their description with "A few weeks ago" in order to investigate the degree to which they could control the tense.

(5) A few weeks ago there was a boy that she - he really wanted to take a swim in-in the sea but he did not have money, so he decided to find some work. And finally he found "staff wanted" on the wall ... (IDN_005_B12)

This excerpt suggests that the learner proficiently controlled both the tense and grammar.

4.4.2 Type B vocabulary (for speech maintenance). Type B vocabulary includes a set of words mainly used in CV by CHN, IDN, THA and TWN learners at varying proficiency levels. It is characterized by the following words: (1) interjections used to fill pauses and keep a conversation going (yeah, yes), (2) hedge terms used to soften claims and fill pauses (some, [I] think, [I] know), (3) amplifiers that inflate claims (just, very, more) and (4) conjunctions that extend the sentences (because, when). When used during the CV tasks, these words help learners having expressive difficulties to maintain their speech.

The quotes below show utterances that $\mathrm{CHN}$ and TWN learners made during the QA session conducted immediately after the non-smoking PD task.

(6) Umm, yes, because I think she is brave because, umm, when you meet some - somesome people like this man, you will be angry ... (CHN_005_B2)

(7) Yeah, in - and I think we - I'm very enjoyed in the restaurant when we are, uh - uh when we - it's together and just together (TWN_043_B2)

\begin{tabular}{lllll}
\hline & Type A & Type B & Type C & Type D \\
\hline L1 & CHN/IDN/ & CHN/IDN/THA/TWN (4), ENS & JPN (8), CHN/IDN/THA/TWN & THA(4), \\
& TWN(2), ENS & (4), ENS & B2/B1_1/2 (7), A2 (6) & JPN/KOR (3) \\
Proficiency & B2/B1_2 (3) & B2 (5), A2 (4) & RP (24) & PD (14) \\
Task & PD (10) & CV (20) & but, can, do, don, for, have, I, if, go, so, to \\
Words & a, and, in, that, & are, because, it, just, know, like, & gor, \\
& the, there, was, & more, no, of, one, or, s, some, think, & is, me, my, not, people, this, & \\
& with & they, very, when, yeah, yes & time, want, we, you &
\end{tabular}

Note(s): Numbers in parentheses represent the number of variables (samples)

Vocabulary

usage among Asian EFL learners

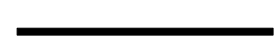


These samples show that learners made efforts to continue speaking by inserting various filler-like terms and conjunctions, many of which are semantically unneeded.

4.4.3 Type Cvocabulary (for negotiation and persuasion). Type $\mathrm{C}$ vocabulary includes a set of words mainly used in RP by JPN learners at varying proficiency levels. It is characterized by words such as (1) first-person pronouns used to show the speaker's will (I, me, my, we), (2) verbs and auxiliary verbs connoting the speaker's desires and abilities (can, want), (3) secondperson pronouns used when persuading the interlocutor (you) and (3) negation terms to refute claims made by the interlocutor (not, do not, but). Here, frequent use of these words during the RP task implied that learners attempted to make a claim, negotiate with their interlocutor and persuade them to accept their requests.

The quote below is from an utterance from a JPN learner during the RP task, in which he/ she tried to explain why he/she requested a refund.

(8) Umm. Your restaurant uh is uh umm $I$ went to your restaurant, but uh the customers smokes very much, so I cannot eat meal. So, please give $m e$ uh give $m e$ back money. I want to eat meal but it's not delicious .... (JPN_025_B1_1)

This sample suggests that the learner tried to make claims and negotiate with the interlocutor by repeatedly using first- and second-person pronouns as well as negation terms.

4.4.4 Type D vocabulary (for novice learners). Finally, Type D vocabulary includes a set of words mainly used in PD by THA learners at the novice or lower intermediate levels. Both Type A and Type D concerned PD, but the latter was linked to vocabulary usage among novice learners who could not appropriately control the tense or grammar. Type D vocabulary includes (1) inappropriate present-tense verbs (e.g. go in place of went) and (2) conjunctions often used as fillers $(s o)$. These words suggest relatively weaker control of tense and logical connections in utterances.

The passages below are PD utterances made by THA learners.

(9) ... He have money. He gone - he go sea ... (THA_014_B1_2)

(10) ... he does not have enough money, mmm, for travel, yeah. So, but he saw the, uh, advertising of the job, and he think ... (THA_008_A2)

The differences in proficiency are clearer in PD than in the other speaking tasks. In PD, learners could speak freely while setting a comfortable pace. Therefore, intermediate or higher-level learners did not make many errors in tense or grammar, as we have already seen in (5), while less proficient learners often lost control of their grammar, even in PD, as suggested in (9) and (10). Although the analysis showed that the task type was the key factor determining the data classification, it may be partly because the task type indirectly highlighted the difference in learners' L2 proficiency.

\section{Conclusion}

Using a newly compiled corpus module consisting of utterances made by Asian learners during L2 English interviews, this study examined how Asian EFL learners' L1s, their L2 proficiency levels and speech task types affected four aspects of vocabulary usage.

With regard to RQ1 (Learner/ENS Gap), we found that (1) gaps were recognized in terms of TKN, STTR and MSL in CV and RP, (2) gaps were hardly seen in terms of MWL and (3) gaps were seen less clearly in PD.

With regard to RQ2 (L1 Group Effect), we found that (1) effects were recognized in terms of TKN and MSL in all task types, (2) effects were seen in terms of STTR in PD and RP, (3) no significant effects were seen in terms of MWL, (4) IDN learners tended to speak more by using slightly longer words and (5) KOR learners tended to use a greater variety of vocabulary but produce shorter sentences. 
Next, with regard to RQ3 (L2 Proficiency Effect), we found that (1) effects were recognized in terms of TKN, STTR and MSL in all task types, (2) advanced learners spoke 36-44\% more, used a $10 \%$ greater variety of words and made $42-67 \%$ longer sentences than novice learners, (3) no significant effects were seen in terms of MWL and (4) the proficiency effect was more significant than the L1 group effect.

Finally, regarding RQ4 (Classification), we found that (1) high-frequency vocabulary usage was influenced more strongly by the task type than either L1s or L2 proficiency level, and (2) vocabulary usage patterns could be classified into four types: Type A vocabulary for grammar control (articles, prepositions, conjunctions, past-tense verbs and existential there), Type B vocabulary for speech maintenance (interjections, hedges and amplifiers), Type $\mathrm{C}$ vocabulary for negotiation and persuasion (negation, first- and second-person pronouns and verbs/auxiliary verbs) and Type D vocabulary for novice learners (inappropriately chosen present-tense verbs and filler-like conjunctions).

These findings provide clues for better understanding L2 English vocabulary usage among Asian learners during speech. Our results also have pedagogical implications. The data analysis proved that the task type influenced vocabulary usage more strongly during speech, which suggests that teachers may need to provide learners with a greater variety of speech tasks. In particular, this should include combined monologue and dialogue tasks, tasks with and without planning time, and tasks based on familiar and/or public topics. We also found that L1s and L2 proficiency levels affected vocabulary usage to some extent. Considering this point, teachers should pay more attention to learner backgrounds when teaching and/or developing relevant teaching materials. Presumably, tailor-made teaching based on custom materials will eventually be needed to develop communicative skills among Asian EFL learners, most of whom do not have ample experience with English for concrete purposes or in natural communication settings. Our findings also point to the existence of four major types of L2 English vocabulary usage among Asian EFL learners, which constitutes a good starting point for considering the design approach to tailor-made teaching.

This study had several limitations. First, we did not examine speech among L2 learners in the ESL area. Here, a comparison between EFL and ESL learners in Asia would offer a clearer picture of L2 English vocabulary usage. Second, we did not consider the possible effects from the interviewers. As Götz (2019) suggested, the interviewer-interviewee relationship may influence the oral performance of learners. This makes it necessary to carefully consider interviewer-related parameters such as gender, age and number of utterances when examining learner speech. Third, the sample numbers were not equal across the four proficiency levels. In particular, the lack of A2-level Korean learners in the current data set might have slightly influenced the results. The author plans to expand the corpus to improve the data balance and coverage of target learners. Finally, we were not able to further investigate the reason for the L1 group effect. For example, it is still unclear why IDN learners spoke more in most task types. This may be the result of the linguistic feature of Indonesian, or it may reflect the culture or the English education system in the country. To discuss this point, it is necessary to compare different learner groups with the same L1 backgrounds. As the ICNALE SD includes data from learners in Hong Kong, the author plans to compare learners in mainland China, Taiwan and Hong Kong. This should help clarify the delicate interface between the L1 group effect and L1 effect. All listed limitations will receive further scrutiny and consideration in future studies.

\section{References}

Bell, C.D.S. (2003), "L2 speech rate in monologic and dialogic activities", Revista Linguagem and Ensino, Vol. 6 No. 2, pp. 55-79.
Vocabulary usage among Asian EFL learners

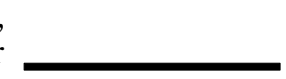


De Jong, N.H., Steinel, M.P., Florijn, A.F., Schoonen, R. and Hulstijn, J.H. (2012), "Facets of speaking proficiency", Studies in Second Language Acquisition, Vol. 34 No. 1, pp. 5-34, doi: 10.1017/ S0272263111000489.

Foster, P. (2020), "Oral fluency in a second language: a research agenda for the next ten years", Language Teaching, Vol. 53 No. 4, pp. 446-461, doi: 10.1017/S026144482000018X.

Gilanlioglu, I. (2002), "The effects of task features on lexis and grammar in L2 oral performance", Unpublished doctoral dissertation, The University of London, Institute of Education.

Götz, S. (2013), Fluency in Native and Nonnative English Speech, John Benjamins Publishing, Amsterdam, Philadelphia.

Götz, S. (2019), "Do learning context variables have an effect on learners' (dis)fluency? Languagespecific vs. universal patterns in advanced learners' use of filled pauses", in Degand, L., Gilquin, G., Meurant, L. and Simon, A.C. (Eds), Fluency and Disfluency across Languages and Language Varieties. Corpora and Language in Use: Proceedings, Presses Universitaires de Louvain, Vol. 4, pp. 177-196.

Granger, S. (1996), "From CA to CIA and back: an integrated contrastive approach to computerized bilingual and learner corpora", in Aijmer, K., Altenberg, B. and Johansson, M. (Eds), Languages in Contrast. Text-Based Cross-Linguistic Studies, Lund University Press, pp. 37-51.

Granger, S. (Ed.), (1998), Learner English on Computer, Longman, London.

Granger, S. (2015), "Contrastive interlanguage analysis: a reappraisal”, International Journal of Learner Corpus Research, Vol. 1 No. 1, pp. 7-24.

Guerrero, R.G. (2004), “Task complexity and L2 narrative oral production”, Unpublished doctoral dissertation, Universitat de Barcelona.

Hasselgren, A. (1994), "Lexical teddy bears and advanced learners: a study into the ways Norwegian students cope with English vocabulary", International Journal of Applied Linguistics, Vol. 4, pp. 237-258.

Hsieh, C.-N. and Wang, Y. (2019), "Speaking proficiency of young language students: a discourseanalytic study", Language Testing, Vol. 36 No. 1, pp. 27-50, doi: 10.1177/0265532217734240.

Ishikawa, S. (2019), "The ICNALE spoken dialogue: a new dataset for the study of Asian learners' performance in L2 English interviews", English Teaching, Vol. 74 No. 4, pp. 153-177.

Ishikawa, S. (2020), "Who speaks more, who speaks less? The amount of speech of Asian learners of English in three kinds of interview tasks", Learner Corpus Studies in Asia and the World, Vol. 4, pp. 110-124.

Liu, Y. (2020), "Relating lexical access and second language speaking performance", Languages, Vols 2020-5 No. 2, p. 13, doi: 10.3390/languages5020013.

MacIntyre, P.D., Dörnyei, Z., Clément, R. and Noels, K.A. (1998), "Conceptualizing willingness to communicate in a L2: a situational model of L2 confidence and affiliation", The Modern Language Journal, Vol. 82 No. 4, pp. 545-562.

Mairano, P. and Santiago, F. (2020), "What vocabulary size tells us about pronunciation skills: issues in assessing L2 learners", Journal of French Language Studies, Vol. 30 No. 2, pp. 141-160, doi: 10. 1017/S0959269520000010.

Segalowitz, M. (2010), Cognitive Bases of Second Language Fluency, Routledge, New York, Abingdon.

Skehan, P. (2009), "Modelling second language performance: integrating complexity, accuracy, fluency, and lexis”, Applied Linguistics, Vol. 30 No. 4, pp. 510-532.

Teng, H.-C. (2007), “A study of task type for L2 speaking assessment”, Paper presented at the Annual Meeting of the International Society for Language Studies (ISLS), Honolulu, Hawaii, Apr 2-4, 2007.

Van Os, M., de Jong, N.H. and Bosker, H.R. (2020), "Fluency in dialogue: turn-taking behavior shapes perceived fluency in native and nonnative speech", Language Learning, Vol. 70 No. 4, pp. 1183-1217, doi: 10.1111/lang.12416. 
Williams, K. (2020), "Exploring perceptions of second language speech fluency through developing and piloting a rating scale for a paired conversational task", Unpublished doctoral dissertation, Carleton University.

\section{Corresponding author}

Shin'ichiro Ishikawa can be contacted at: iskwshin@gmail.com

For instructions on how to order reprints of this article, please visit our website: www.emeraldgrouppublishing.com/licensing/reprints.htm Or contact us for further details: permissions@emeraldinsight.com 